\title{
Aminosalicylic acid reduces ER stress and Schwann cell death induced by MPZ mutations
}

\author{
EUN HYUK CHANG ${ }^{1 *}$, WON MIN MO ${ }^{2 *}$, HYUN MYUNG DOO ${ }^{3}$, JI-SU LEE $^{3}$, \\ HWAN TAE PARK ${ }^{4}$, BYUNG-OK $\mathrm{CHOI}^{2,3}$ and YOUNG BIN HONG ${ }^{5}$
}

\begin{abstract}
${ }^{1}$ Samsung Biomedical Research Institute, Samsung Advanced Institute of Technology, Samsung Electronics Co., Ltd.;
${ }^{2}$ Department of Neurology, Samsung Medical Center, Sungkyunkwan University School of Medicine, Seoul 06351; ${ }^{3}$ Department of Health Sciences and Technology, SAIHST, Sungkyunkwan University, Seoul 03063;

Departments of ${ }^{4}$ Physiology and ${ }^{5}$ Biochemistry, College of Medicine, Dong-A University, Busan 49201, Republic of Korea
\end{abstract}

Received December 14, 2018; Accepted April 25, 2019

DOI: $10.3892 /$ ijmm.2019.4178

\begin{abstract}
Mutations in myelin protein zero (MPZ) cause inherited peripheral neuropathies, including Charcot-Marie-Tooth disease (CMT) and Dejerine-Sottas neuropathy. Mutant MPZ proteins have previously been reported to cause CMT via enhanced endoplasmic reticulum (ER) stress and Schwann cell (SC) death, although the pathological mechanisms have not yet been elucidated. In this study, we generated an in vitro model of rat SCs expressing mutant MPZ (MPZ V169fs or R98C) proteins and validated the increase in cell death and ER stress induced by the overexpression of the MPZ mutants. Using this model, we examined the efficacy of 3 different aminosalicylic acids (ASAs; 4-ASA, sodium 4-ASA and 5-ASA) in alleviating pathological phenotypes. FACS analysis indicated that the number of apoptotic rat SCs, RT4 cells, induced by mutant MPZ overexpression was significantly reduced following treatment with each ASA. In particular, treatment with 4-ASA reduced the levels of ER stress markers in RT4 cells induced by V169fs MPZ mutant overexpression and relieved the retention of V169fs mutant proteins in the ER. Additionally, the level of an apoptotic signal mediator ( $\mathrm{p}-\mathrm{JNK}$ ) was only decreased in the RT4 cells expressing R98C MPZ mutant protein following treatment with 4-ASA. Although 4-ASA is known as a free
\end{abstract}

Correspondence to: Dr Byung-Ok Choi, Department of Neurology, Samsung Medical Center, Sungkyunkwan University School of Medicine, 81 Irwon-ro, Gangnam-gu, Seoul 06351, Republic of Korea E-mail: bochoi77@hanmail.net

Dr Young Bin Hong, Department of Biochemistry, College of Medicine, Dong-A University, 32 Daesin Gongwon-ro, Seo-gu, Busan 49201, Republic of Korea

E-mail: youngbinhong@gmail.com

${ }^{*}$ Contributed equally

Key words: myelin protein zero, endoplasmic reticulum stress, Schwann cell death, reactive oxygen species, Charcot-Marie-Tooth disease radical scavenger, treatment with 4-ASA in the in vitro model did not moderate the level of reactive oxygen species, which was elevated by the expression of mutant MPZ proteins. On the whole, the findings of this study indicate that treatment with 4-ASA reduced the ER stress and SC death caused by 2 different MPZ mutants and suggest that ASA may be a potential therapeutic agent for CMT.

\section{Introduction}

Myelin protein zero (MPZ) is one of the components of myelin in Schwann cells. It comprises $21 \%$ of the protein in the sheath of peripheral nerves, and is responsible for ensheathment and axonal protection by linking adjacent lamellae to stabilize the myelin assembly (1-3). There are $>200$ known MPZ mutations that cause dominantly inherited peripheral neuropathies, known as Charcot-Marie-Tooth disease type 1B (CMT1B), congenital hypomyelinating neuropathy 2 (CHN2), or Dejerine-Sottas neuropathy (DSN; V169fs) $(4,5)$. CMT1B is a demyelinating neuropathy resulting in distal muscle atrophy and DSN is a more severe form of CMT with a childhood-onset. The symptoms of CHN2 include respiratory difficulty, muscle weakness and incoordination, areflexia and ataxia (6). These mutants also cause heterogeneous neuropathies through diverse pathological mechanisms due to their different gain-of-function traits, which are not yet fully understood (7).

According to previous studies on the pathological mechanisms of MPZ mutations, mutant MPZ proteins commonly cause CMT1B by inducing endoplasmic reticulum (ER) stress, which is mediated by the unfolded protein response (UPR) (8-12). ER stress is initiated when unfolded proteins accumulate and the production of reactive oxygen species (ROS) is induced through oxidative protein folding. Once the concentration of unfolded proteins exceeds the capacity of a chaperone protein, binding immunoglobulin protein (BiP), these surplus proteins activate ER membrane-bound proteins, such as the N-terminal ends of pancreatic ER kinase RNA-like ER kinase (PERK), inositol-requiring enzyme $1 \alpha($ IRE1 $\alpha)$ and activating transcription factor 6 (ATF6) (13). Activated PERK, IRE1 $\alpha$ and ATF6 initiate the apoptotic cascade, which is 
initially mediated by pro-apoptotic proteins, including C/EBP homologous protein (CHOP), c-Jun N-terminal kinase (JNK) or microRNAs (miRNAs or miRs) $(14,15)$.

Amino derivatives of salicylic acids [aminosalicylic acids (ASAs)] are safe drugs that are commonly used in clinical practice. In particular, 4-ASA, one of the most effective antibiotics, has been used for the treatment of tuberculosis since 1944 (16). In addition, 4- and 5-ASA can be used for the treatment of inflammatory bowel disease owing to their anti-inflammatory properties (17). Further mechanistic studies have indicated that 4- and 5-ASA act to scavenge free radicals that produce inflammation $(18,19)$. A previous study also demonstrated that 3-, 4-, and 5-ASA reduce the ROS concentration, thereby relieving manganese neurotoxicity in dopaminergic human neuroblastoma cells (20).

In this study, we generated in vitro models expressing mutant MPZ protein that caused ER stress and Schwann cell death and investigated whether the 3 ASAs can alleviate these pathological effects.

\section{Materials and methods}

Cell culture and transfection. Rat Schwann cells, RT4 cells (RT4-D6P2T, CRL-2768, ATCC), were cultured in high-glucose Dulbecco's modified Eagle's medium (DMEM; Biowest) containing $10 \%$ fetal bovine serum and $1 \%$ penicillin-streptomycin (Biowest) at $37^{\circ} \mathrm{C}$ in a $5 \% \mathrm{CO}_{2}$ atmosphere. The MPZ gene was amplified from the pCMV6-entry-MPZ vector (Origene). The amplified PCR product was cloned into the pCMV-Myc or p-EGFP(C1) vector (Clontech). Mutant genes (V169fs, L184fs, R185fs, S226fs and R98C) were generated using the QuikChange Site-Directed Mutagenesis kit (Stratagene). To express wild-type MPZ and mutant MPZ genes, the RT4 cells $\left(2 \times 10^{5}\right)$ seeded on 6-well culture plates were transfected with MPZ-containing vectors [pCMV-Myc-MPZ WT/V169fs/R98C and pEGFP(C1)-MPZ WT/V169fs], as well as their control vectors [pCMV-Myc and pEGFP(C1)] using Lipofectamine 3000 (Invitrogen) according to the manufacturer's instructions. Based on western blot analysis and immunocytochemistry, the transcription efficiency was $>90 \%$. The transfected cells were incubated at $37^{\circ} \mathrm{C}$ for $48 \mathrm{~h}$. The RT4 cells $\left(2 \times 10^{5}\right)$ were transfected with the MPZ expression vectors and treated with the drugs (1-100 $\mu \mathrm{M})$ [para(4)-aminosalicylic acid (4-ASA), sodium 4-aminosalicylic acid (s4-ASA) and 5-aminosalicylic acid (5-ASA)] (Sigma-Aldrich), as well as their solvent (PBS) solution as a negative control for $24 \mathrm{~h}$. The direct counting of cell numbers was performed after the collected cells were stained with trypan blue (T8154, Sigma-Aldrich). Ten microliters of cells were mixed with an equal volume of Trypan blue then cells were immediately counted with a hemacytometer (Sigma-Aldrich, Z359626) under a microscopy

Fluorescence-activated cell sorting (FACS) for the measurement of cell death. In order to measure cell death, the RT4 cells were seeded in 6-well culture plates at a density of $2 \times 10^{5}$ cells per well. The RT4 cells were transiently transfected with wild-type MPZ or mutant MPZ for $24 \mathrm{~h}$, then treated with the ASA compounds (1-100 $\mu \mathrm{M})$ for $24 \mathrm{~h}$ and harvested. Annexin V fluorescein isothiocyanate (FITC) and propidium iodide (PI) staining (BD Biosciences Pharmingen) was performed by incubating the cells in the dark for $15 \mathrm{~min}$ at room temperature in binding buffer (10 mM HEPES, $140 \mathrm{mM}$ $\mathrm{NaCl}$, and $2.5 \mathrm{mM} \mathrm{CaCl}_{2}$, at $\mathrm{pH}$ 7.4) saturated with Annexin $\mathrm{V}$ FITC and PI. Following incubation, the cells were washed with cold PBS, pelleted and analyzed by a FACS VERSE analyzer (BD Biosciences). We determine the number of live, apoptotic and necrotic cells by counting the numbers of Annexin $\mathrm{V}^{-} / \mathrm{PI}^{-}$cells, Annexin $\mathrm{V}^{+} / \mathrm{PI}^{-}$cells, and Annexin $\mathrm{V}^{+} / \mathrm{PI}^{+}$ cells, respectively.

DCFDA assay. The control experiment involved the treatment of the RT4 cells with Brefeldin A (Sigma-Aldrich) $(5 \mu \mathrm{M})$ for $48 \mathrm{~h}$. For the experimental groups, the RT4 cells were transfected with MPZ V169fs or R98C mutant vectors for $48 \mathrm{~h}$ with the ASA compounds (1-100 $\mu \mathrm{M})$ or ascorbic acid (A92902, Sigma-Aldrich). To measure ROS levels in the control and experimental groups, the $\mathrm{H}_{2}$ DCFDA assay was performed as per the manufacturer's recommendations (Invitrogen). Initially, the RT4 cells were harvested and incubated with $10 \mu \mathrm{M}$ $\mathrm{H}_{2}$ DCFDA at $37^{\circ} \mathrm{C}$ for $40 \mathrm{~min}$. After being chilled on ice, the cells were pelleted and analyzed with a FACS VERSE analyzer (BD Biosciences). The fluorescence intensity of $\mathrm{H}_{2}$ DCFDA formed by the reaction between $\mathrm{H}_{2}$ DCFDA and intracellular ROS in $>10,000$ viable cells per sample was analyzed at an excitation wavelength of $488 \mathrm{~nm}$ and an emission wavelength of $525 \mathrm{~nm}$ using a FACS VERSE analyzer (BD Biosciences). The experiments were repeated at least 3 times, and the most representative histogram data are presented.

Western blot analysis. The expression of MPZ proteins and ER stress markers was confirmed using a standard western blot analysis. Total cells were harvested and lysed with RIPA lysis buffer (Thermo Fisher Scientific). The cell lysates were centrifuged at $13,000 \mathrm{xg}$ for $15 \mathrm{~min}$ at $4^{\circ} \mathrm{C}$, and the supernatants were used for quantification using bicinchoninic acid (BCA) method. A total of $20 \mu \mathrm{g}$ of protein was separated and transferred onto polyvinylidene fluoride (PVDF) membranes. The membranes were then stained with $0.1 \%$ Ponceau S solution (Sigma-Aldrich) to ensure equal loading of the samples, and non-specific binding was blocked with a blocking buffer (Casein blocking buffer 10X; Sigma-Aldrich) for $1 \mathrm{~h}$ at room temperature. The membranes were incubated overnight at $4^{\circ} \mathrm{C}$ with the following primary antibodies: Anti-Myc-tag (1:2,000; ab9106, Abcam), anti-binding immunoglobulin protein (BiP, \#3177), anti-C/EBP-homologous protein (CHOP, \#5554), anti-cleaved caspase-3 (Asp175, \#9661), anti-phospho-AKT (ser473, \#9271), anti-phospho-SAPK/JNK (Thr183/Tyr185, \#9251) (all 1:1,000) (all from Cell Signaling Technology) and anti- $\beta$-actin (A2228, Sigma-Aldrich). Subsequently, bound antibodies were visualized with anti-mouse (\#7076) or anti-rabbit IgG HRP-linked secondary antibodies (\#7074) (all 1:2,000) (all from Cell Signaling Technology) by incubating $1 \mathrm{~h}$ at room temperature and Western blotting luminol reagent (Santa Cruz Biotechnology) in order to detect proteins. The integrated optical densities of the immunoreactive protein bands were measured using Image J software (https://imagej.nih.gov).

Immunocytochemistry and confocal imaging. For immunofluorescence staining, the RT4 cells $\left(2 \times 10^{4}\right)$ were seeded on 
coverslips in 24-well culture plates. After being transfected with wild-type MPZ or mutant MPZ (pCMV-Myc-MPZ WT, V169fs, R98C and pEGFP-MPZ WT, V169fs) for $24 \mathrm{~h}$, the cells were treated with the drugs ( $100 \mu \mathrm{M}$ of 4-, s4-, or 5-ASA) for $24 \mathrm{~h}$. Subsequently, they were fixed in $4 \%$ paraformaldehyde for 20 min, washed with PBS for 5 min 3 times, and blocked with $0.1 \%$ Triton X-100 in 5\% normal goat serum for $1 \mathrm{~h}$ at room temperature. Fixed cells were incubated with the primary antibodies, anti-Myc-tag (1:500; ab9106, Abcam) and anti-protein disulfide isomerase (PDI; 1:100; \#2446, Cell Signaling Technology), overnight at $4^{\circ} \mathrm{C}$. After being washed 3 times with PBS for 5 min each time, the cells were incubated with the appropriate secondary antibodies, including Alexa Fluor 488 goat anti-rabbit $\operatorname{IgG}(1: 1,000$, A11008) and Alexa Fluor 568 goat anti-mouse $\operatorname{IgG}(1: 1,000$, A11001) (Molecular Probes) for $1 \mathrm{~h}$ at room temperature. The cells were finally washed and mounted in Vectashield hardset antifade mounting medium (Vector Laboratories) with 4,6-diamidino-2-phenylindole (DAPI) to allow for the visualization of the nuclei. The stained sections were visualized under a laser scanning confocal microscope (CLSM700; Carl Zeiss).

Statistical analysis. All experiments (western blot analysis, FACS, immunocytochemistry and cell quantification) were performed at least 3 independent times. We tested for and found normal distributions and equal variances in our sample distributions. To determine the statistical significance within the multiple groups in western blot analysis and cell quantification, we used one-way ANOVA with a post hoc Tukey's multiple comparison test to confirm whether the $\mathrm{F}$ value was greater than F-critical value. For comparisons between the groups (control vs 4-ASA treated cells after transfection with either MPZ-V169fs or R98C vectors), Student's $\mathrm{t}$-tests were used to determine the effects of 4-ASA in FACS analysis of Annexin $\mathrm{V}^{-} / \mathrm{PI}^{-}$cells, Annexin $\mathrm{V}^{+} / \mathrm{PI}^{-}$cells, and Annexin $\mathrm{V}^{+} / \mathrm{PI}^{+}$cells. $\mathrm{P}<0.05$ was considered statistically significant.

\section{Results}

MPZ mutations cause Schwann cell death. Previous studies on 2 MPZ mutants (V169fs and R98C) indicated that these mutants induce Schwann cell death and the elevation of ER stress in vitro and in vivo $(10,21)$. In particular, the R98C mutant has been reported to activate the IRE1 pathway, leading to apoptosis in vivo (21). The V169fs mutant has also been shown to induce ER stress and cell death by being retained in the ER compartments of non-Schwann cells in vitro (HeLa or 293 cell lines) (10).

To validate the induction of Schwann cell death or ER stress by MPZ mutant proteins, we generated wild-type (WT) and 5 mutant MPZ (V169fs, L184fs, R185fs, S226fs or R98C) expression vectors by site-directed mutagenesis. From western blot analysis and immunocytochemistry, we confirmed the effective expression of MPZ proteins by the transient transfection of WT and mutant MPZ vectors into the RT4 cells with a $>90 \%$ transfection efficiency (Figs. 1, 2 and S1). In addition, we observed that the levels of ER stress markers, such as BiP and CHOP were altered by either MPZ-V169fs or MPZ-R98C overexpression. The CHOP expression levels were elevated by the overexpression of MPZ-V169fs and MPZ-R98C mutants, while the BiP level was elevated only by the overexpression of MPZ-V169fs mutant (Fig. S1). Thus we proceeded with further experiments using only the MPZ-V169fs and MPZ-R98C mutant.

To determine whether the overexpression of two MPZ mutants (V169fs and R98C) affects Schwann cell viability, we transfected $2 \mathrm{MPZ}$ mutant-overexpressing vectors into the RT4 cells. Within $48 \mathrm{~h}$, the detachment of transfected RT4 cells from the bottom of the dish was observed. Manual cell counting following Trypan blue staining indicated that the overexpression of the V169fs or R98C mutant in the RT4 cells caused 65 or $32 \%$ significant cell death compared to the expression of wild-type protein, respectively (Fig. 1A). To validate this result, the number of Annexin $\mathrm{V}^{-}$and PI-positive RT4 cells following transfection with the MPZ mutants were measured by FACS analysis. Expectedly, the percentage of live RT4 cells indicated a 38\% significant decrease following the expression of the V169fs mutant compared to wild-type MPZ (Fig. 1B). This cell death was driven by the significant induction of apoptosis. On the other hand, the expression of the $\mathrm{R} 98 \mathrm{C}$ mutant resulted in a $46 \%$ significant reduction in the late apoptotic/dead cell population; however, the number of live cells was not significantly altered. This result was further confirmed by western blot analysis. The expression of the V169fs mutant led to a 2 -fold $(\mathrm{P}<0.05)$ increase in cleaved caspase- 3 levels $48 \mathrm{~h}$ following transfection compared to the wild-type control (Fig. 1C).

ER stress and ROS levels are elevated in Schwann cells by the expression of MPZ mutants. As previously reported, selected MPZ mutant proteins (S22_W28 deletion, V169fs, 550del3insG, S63del and R98C) induce ER stress in vitro or in vivo $(7,10,21-23)$. In this study, to determine whether Schwann cell death is associated with ER stress in the mutant MPZ protein (V169fs or R98C)-expressing cells, the levels of $\mathrm{BiP}$ and CHOP were measured in cell lysates of transfected RT4 cells (Fig. 2A and Fig. S1). The BiP level exhibited a 1.3-fold significant increase in the MPZ V169fs-expressing RT4 cells, whereas the overexpression of the R98C mutant did not markedly alter the BiP levels. The level of CHOP was significantly increased in the cells overexpressing either MPZ mutant (V169fs or R98C) compared to the cells overexpressing the wild-type control.

To link the elevation of ER markers to the accumulation of mutant protein in the ER, we assessed the localization of MPZ (wild-type/mutant) proteins in RT4 cells. We detected MPZ with an anti-Myc antibody and marked the ER with anti-PDI in transfected RT4 cells (Fig. 2B). Wild-type and R98C mutant proteins were found throughout the cytosol, whereas V169fs mutant protein co-localized with PDI.

As the retention of unfolded proteins in the ER possibly causes an elevation of oxidative stress levels in the ER $(24,25)$, we also determined the extent of induction of ROS levels using DCFDA, which detects intracellular ROS levels through the fluorometric measurement of DCF oxidation. Using FACS analysis, the ROS levels underwent a statistically significant increase in the cells expressing the V169fs and R98C mutants, respectively (Fig. 2C). On the 
A

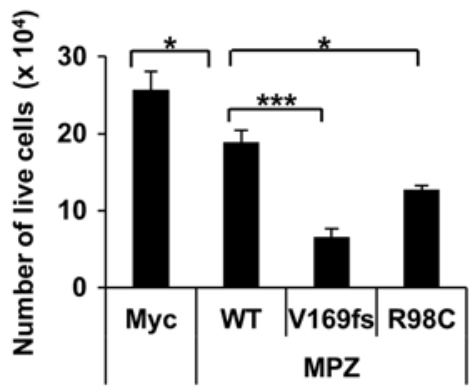

C
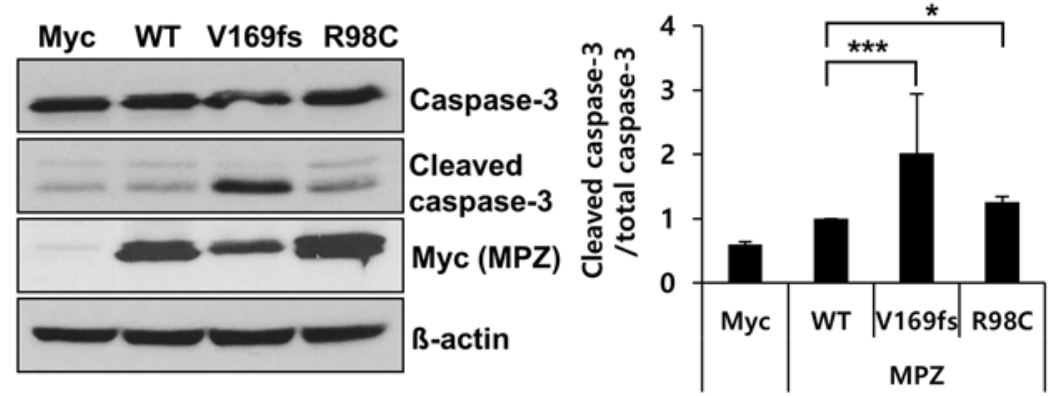

B
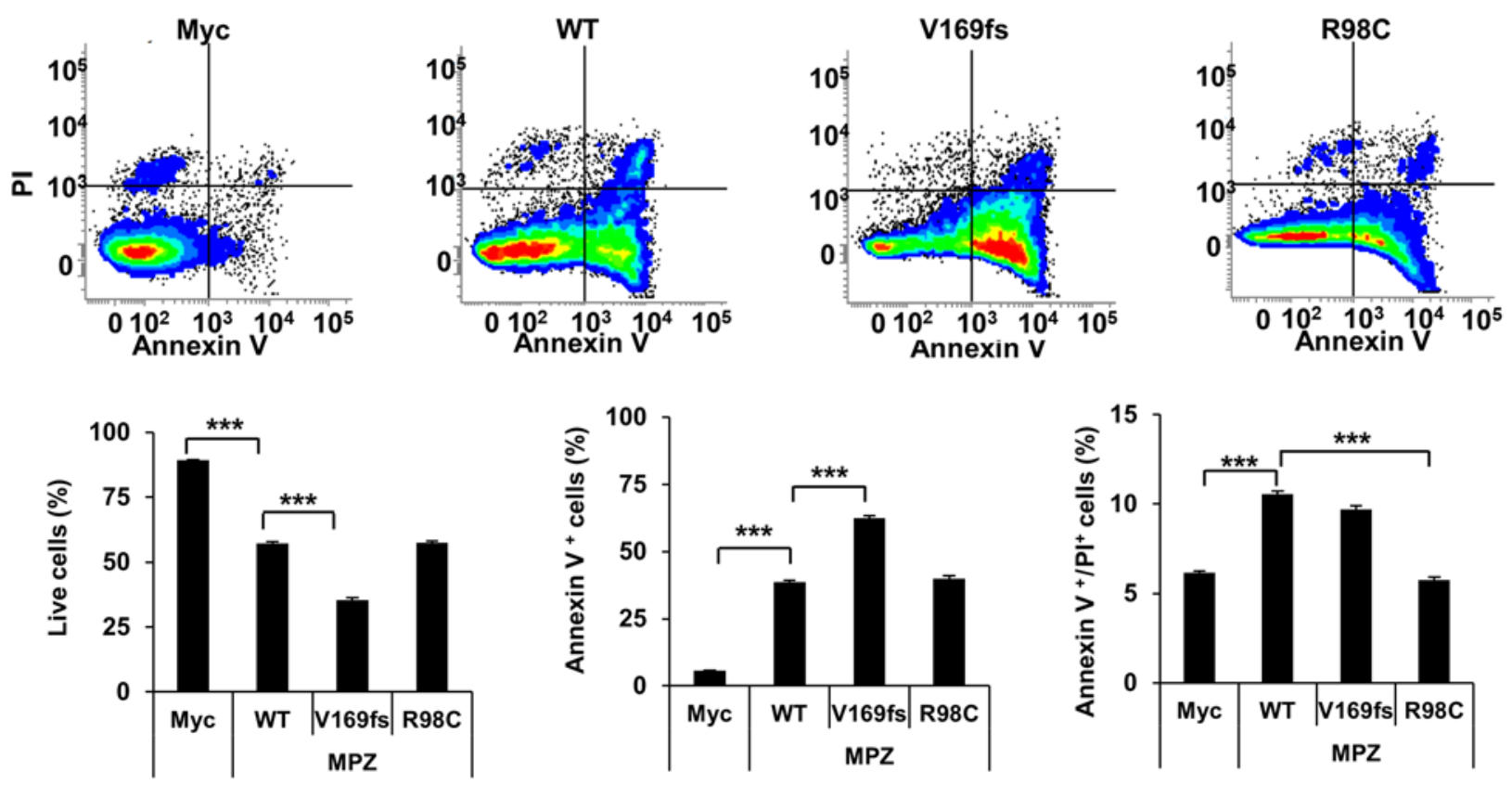

Figure 1. Schwann cell death is induced by MPZ mutant overexpression. (A) Following the overexpression of mutant MPZ proteins (V169fs and R98C) in RT4 cells for $48 \mathrm{~h}$, the number of live rat Schwann cells was reduced $\left(\mathrm{n}=3 ;{ }^{*} \mathrm{P}<0.05 ;{ }^{* * *} \mathrm{P}<0.001\right)$. (B) FACS analysis showing the quantities of live cells, apoptotic cells (Annexin $\mathrm{V}^{+}$cells), and late-stage apoptotic/necrotic cells (Annexin $\mathrm{V}^{+}$and $\mathrm{PI}^{+}$cells) by Annexin $\mathrm{V}$ and $\mathrm{PI}$ staining ( $\mathrm{n}=3$; ${ }^{* * *} \mathrm{P}<0.001$ ). (C) Western blot analysis indicating the levels of cleaved and total caspase-3 following the overexpression of MPZ mutant. Quantification of the western blot analysis data indicating an increase in the level of cleaved caspase-3 following the overexpression of $\mathrm{MPZ}$ mutant ( $=3$; $\left.{ }^{*} \mathrm{P}<0.05 ;{ }^{* * *} \mathrm{P}<0.001\right) . \mathrm{MPZ}$, myelin protein zero.

whole, the protein localization and ER stress levels within the Schwann cells varied depending on the particular MPZ mutation, yet both the V169fs and R98C mutations resulted in Schwann cell death.

ASAs reduce the cell death induced by MPZ mutants. To examine whether ASAs reduce Schwann cell death, we treated the MPZ-transfected RT4 cells with 3 different concentrations $(1,10$, or $100 \mu \mathrm{M})$ of 4 -, s4-, or 5-ASA for $24 \mathrm{~h}$. This treatment resulted in the distinctively less detachment of transfected RT4 cells. Quantitative analysis of the live cells indicated that the number of live cells was significantly increased following treatment with $100 \mu \mathrm{M}$ of ASAs, except for the case of 5-ASA treatment in the R98C MPZ mutant proteins (Fig. 3A). To verify whether the enhancement of cell viability was dependent on the dose of ASAs, we used one-way ANOVA with a post hoc Tukey's multiple comparison test to compare the numbers of live cells following treatment with various concentrations of each ASA (Fig. 3A). Although the ASA-treated group exhibited a significant enhancement of cell viability compared to an experimental control group, this increment was not dose-dependent. FACS analysis of the Annexin V and PI indicated that 4-ASA treatment significantly enhanced the number of live RT4 cells overexpressing either the V169fs (1.24-fold increase from control group) (Fig. 3B) or R98C mutant (1.28-fold increase from control group) (Fig. 3C). The increase in cell survival occurred by virtue of a significant reduction in both apoptosis and necrosis (V169fs: $10.8 \%$ reduction of apoptosis and $51.5 \%$ reduction of necrosis; $\mathrm{R} 98 \mathrm{C}$ : $32.4 \%$ reduction of apoptosis and $13.4 \%$ reduction of necrosis). Treatment with s4- and 5-ASA also increased the number of live cells via a reduction in both apoptosis and necrosis (data not shown). The reduction of the apoptosis of the mutant-overexpressing RT4 cells following 4 -ASA treatment $(100 \mu \mathrm{M})$ was also determined by using cleaved caspase-3 staining (Fig. 3D). Taken together, the results indicated that 4-, s4-, and 5-ASA 
A
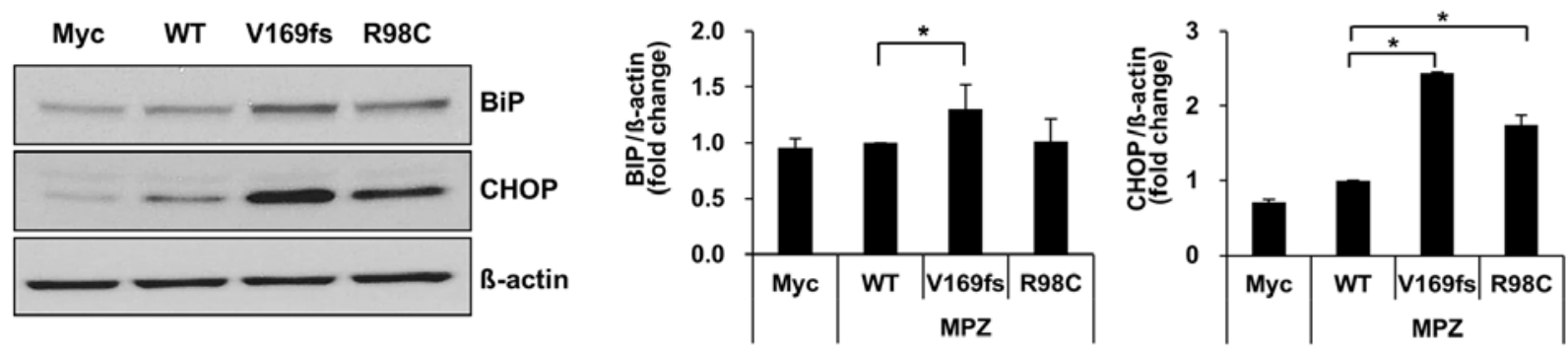

B

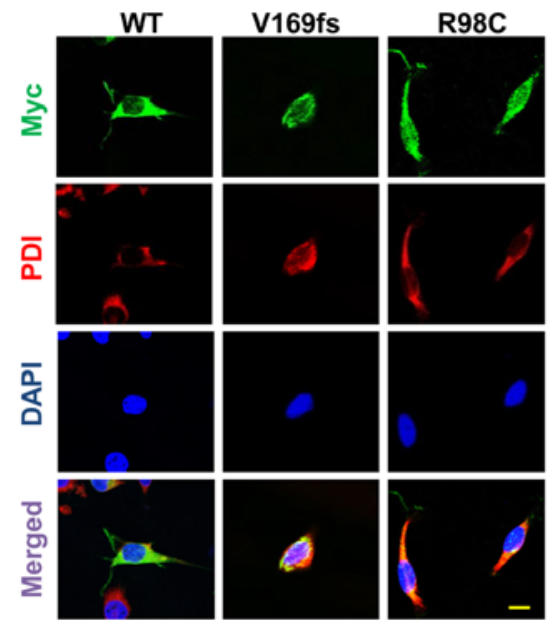

C

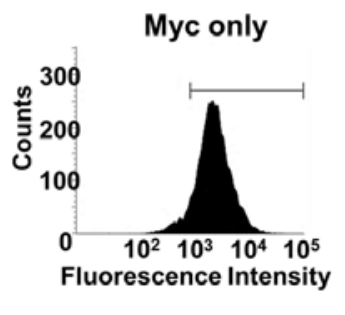

V169fs

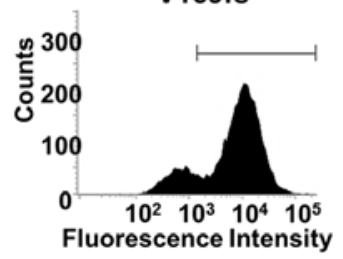

WT

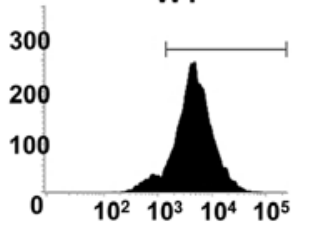

Fluorescence Intensity

R98C

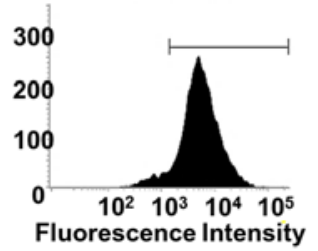

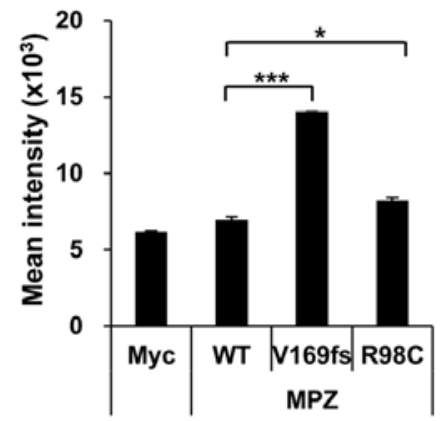

Figure 2. ER stress in Schwann cells is induced by MPZ mutant overexpression. (A) Western blot analysis demonstrating changes in the levels of ER stress markers (BiP and CHOP) following transfection with wild-type, V169fs and R98C mutants of MPZ in RT4 cells. Quantitative analyses of the western blot analysis data were performed for $\mathrm{BiP}$ and $\mathrm{CHOP}$ normalized to $\mathrm{B}$-actin $\left(\mathrm{n}=3\right.$; $\left.{ }^{*} \mathrm{P}<0.05\right)$. (B) Immunocytochemistry for overexpressed wild-type MPZ and the mutants (Myc), and an ER marker (PDI) indicating the localization of MPZ proteins in relation to the ER in RT4 cells (n=3; scale bar, $10 \mu \mathrm{m}$ ). (C) DCFDA assay showing that expression of both mutants resulted in an augmentation of ROS levels in RT4 cells. Expression of the MPZ V169fs and R98C mutants resulted in statistically significant 2 - and 1.2 -fold increases in ROS levels, respectively $\left(\mathrm{n}=3 ;{ }^{*} \mathrm{P}<0.05 ;{ }^{* * * *} \mathrm{P}<0.001\right)$. MPZ, myelin protein zero.

significantly increased Schwann cell viability through a significant reduction in both apoptosis and necrosis.

\section{4-ASA reduces ER stress induced by MPZ mutant proteins.} 4- and 5-ASA are used clinically as anti-inflammatory drugs, particularly for patients with inflammatory bowel disease (26). 4-ASA is recognized as an antibiotic for the treatment of tuberculosis (27). Apart from these known effects of the ASAs, a previous study demonstrated the drug repositioning of ASA in reducing manganese-induced ROS generation and cell death in human neuroblastoma cells (20). In this study, we examined whether 4-, s4-, or 5-ASA can alleviate ER stress in Schwann cells induced by the presence of mutant MPZ proteins. We measured the level of ER stress by detecting BiP and CHOP expression at $24 \mathrm{~h}$ following treatment with the drugs. The $\mathrm{BiP}$ levels in the V169fs-transfected RT4 cells were significantly decreased in response to treatment with $100 \mu \mathrm{M} 4$-ASA (23\% decrease from control) (Fig. 4A). However, the s4- and 5-ASA-treated cells did not exhibit a significant decrease in BiP levels. Similarly, the CHOP level was significantly decreased only in the $100 \mu \mathrm{M}$ 4-ASA-treated V169fs mutant-expressing RT4 cells (37\% decrease from control). On the other hand, the level of neither ER stress marker was significantly decreased in the R98C-transfected RT4 cells following treatment with 4-ASA in comparison with the control, apart from the BiP level in the 5-ASA (10 $\mu \mathrm{M})$-treated group (Fig. 4B). As ER stress in V169fs mutant-transfected RT4 cells occurred due to the retention of mutant MPZ proteins within the ER compartment, we observed the localization of MPZ V169fs protein, which is tagged with EGFP. GFP fluorescence was observed in the cytosol of RT4 cells following treatment with $100 \mu \mathrm{M}$ of ASAs (Fig. 4C). Treatment of the RT4 cells expressing V169fs mutant protein with 4-ASA resulted in a cellular phenotype similar to that of wild-type protein-expressing RT4 cells, in which MPZ protein was distributed throughout Schwann cell processes (yellow arrowheads) rather being restricted to the soma or ER compartment (PDI-positive). Treatment with s4-ASA and 5-ASA also resulted in GFP expression in Schwann cell processes, which were shorter than those of the 4-ASA-treated RT4 cells. Taken together, these results indicated that treatment with $100 \mu \mathrm{M}$ of 4-ASA ameliorated the cellular phenotypes and the ER stress caused by MPZ V169fs mutant protein.

In R98C mutant-expressing cells, no significant changes were observed in the BiP or CHOP levels following treatment with any of the 3 ASAs, apart from the decrease observed in BiP expression with $10 \mu \mathrm{M}$ 5-ASA (Fig. 4B). This result was similar to that of a previous study, which indicated that the ablation of CHOP did not rescue the phenotype of R98C transgenic mice and that the R98C mutant activates the IRE1 pathway of the UPR (21). Subsequently, in order to eludicate the mechanism of apoptosis that results from the overexpression 
A
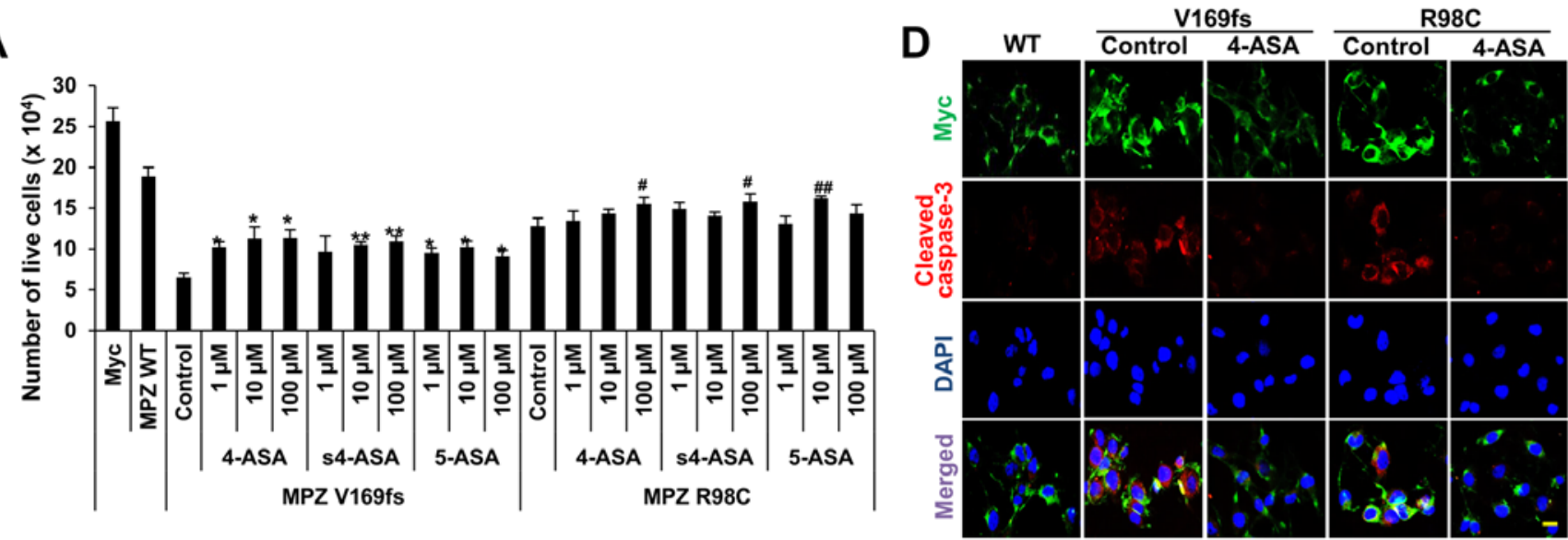

B
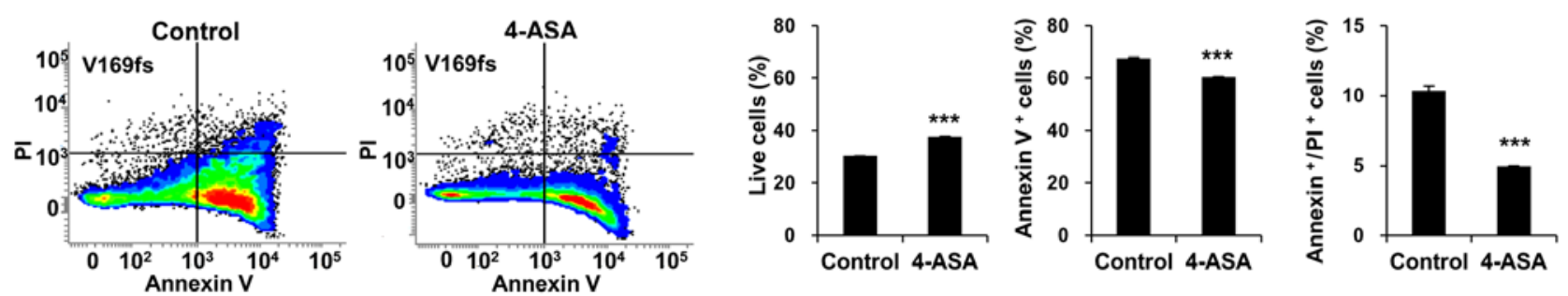

C
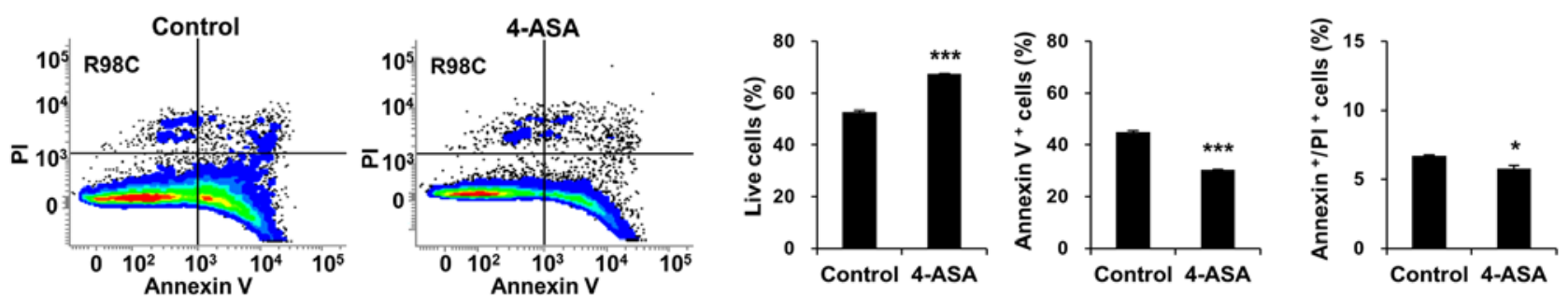

Figure 3. Effects of ASAs on cell death resulting from MPZ mutant expression. (A) Single cell counts indicating that treatment with 4-, s4- or 5-ASAs increased the number of live RT4 cells expressing the V169fs or R98C mutants. An asterisk (") indicates statistical significance vs. MPZ V169fs control. A pound sign $\left({ }^{\#}\right)$ indicates statistical significance vs. MPZ R98C control $\left(\mathrm{n}=3 ;{ }^{*} \mathrm{P}<0.05 ;{ }^{* *} \mathrm{P}<0.01 ;{ }^{*} \mathrm{P}<0.05 ;{ }^{\# \#} \mathrm{P}<0.01\right)$. (B and C) FACS analysis also showing the quantities of live cells, apoptotic cells (Annexin $\mathrm{V}^{+}$cells), and late-stage apoptotic/necrotic cells (Annexin $\mathrm{V}^{+}$and $\mathrm{PI}^{+}$cells) by Annexin $\mathrm{V}$ and PI staining of MPZ V169fs (B) or R98C (C) overexpressing RT4 cells ( $\mathrm{n}=3$; $\left.{ }^{*} \mathrm{P}<0.01 ;{ }^{* * *} \mathrm{P}<0.001\right)$. (D) RT4 cells expressing the wildtype or mutants (V169fs or R98C) were stained with cleaved caspase-3 for detecting the apoptosis of the RT4 cells ( $\mathrm{n}=5$; scale bar, $20 \mu \mathrm{m}$ ). MPZ, myelin protein zero; ASA, aminosalicylic acid.

of MPZ R98C protein in Schwann cells, we detected the levels of phosphorylated JNK (p-JNK) as a marker of ER stress-induced apoptosis (28). As expected, the RT4 cells treated with $100 \mu \mathrm{M}$ 4-ASA and expressing the R98C mutant exhibited a $64 \%$ significant reduction in the p-JNK levels (Fig. 4E), while no significant changes were observed in the cells expressing the V169fs mutant (Fig. 4D). Therefore, 4ASA treatment reduced the level of UPR-mediated pro-apoptotic markers in RT4 cells expressing the R98C MPZ mutant, while ER stress was not affected.

4-ASA does not affect ROS levels induced by MPZ mutant proteins. Increased ER stress may also results in ROS accumulation (29), which is caused by the overexpression of mutant MPZ proteins in RT4 cells. Thus, in this study, we examined whether 4-ASA treatment can alleviate ROS levels. RT4 cells expressing each MPZ mutant were treated with 3 different concentrations of 4-ASA, and the ROS level was measured by DCFDA assay. Notably, none of the 4-ASA-treated groups exhibited any marked changes in ROS levels (Fig. 5A and B). We also used ascorbic acid $(600 \mu \mathrm{M})$ to confirm the results of the DCFDA assay with the MPZ V169fs mutant, as ascorbic acid potently inhibits ROS production $(30,31)$. Treatment with ascorbic acid resulted in a 26.5\% significant reduction in ROS levels (Fig. 5C). These data suggest that 4-ASA does not affect the ROS levels, which are elevated by ER stress caused by the expression of mutant MPZ proteins.

\section{Discussion}

Currently, there is no treatment for CMT that affects the course of the disease. In this study, we investigated whether amino derivatives of salicylic acids (4-, s4-, or 5-ASA) may be effective treatments in rat Schwann cells overexpressing $2 \mathrm{MPZ}$ mutations, V169fs and R98C, which cause DSN and CMT1B, respectively $(4,32)$. We initially generated in vitro models overexpressing the mutant proteins in rat Schwann cells, and found that only treatment with $100 \mu \mathrm{M}$ 4-ASA exhibited significant drug efficacy for those $2 \mathrm{MPZ}$ mutations: i) 4-ASA treatment increased the viability of Schwann cells which overexpressed MPZ V169fs or MPZ R8C mutant proteins; ii) 4-ASA reduced the level of ER stress markers (BiP and CHOP) in MPZ 
A

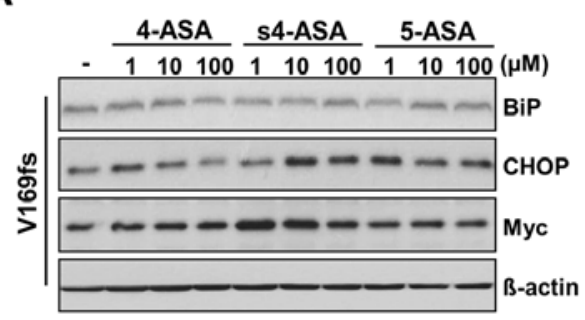

B

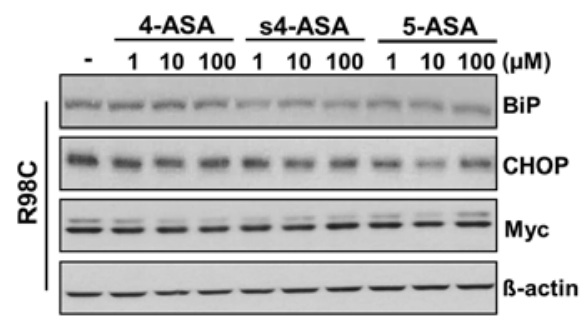

C

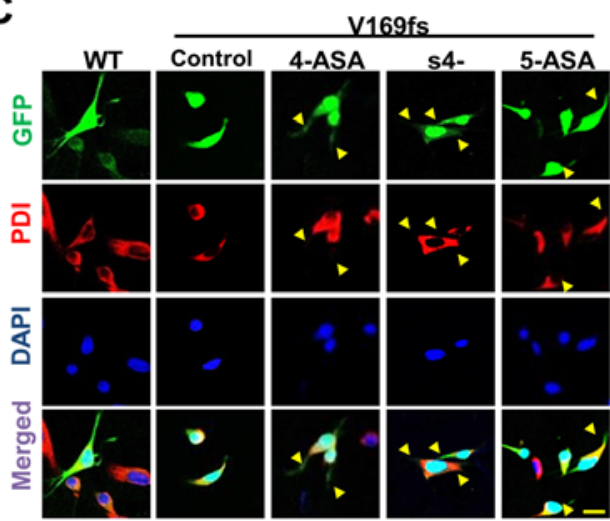

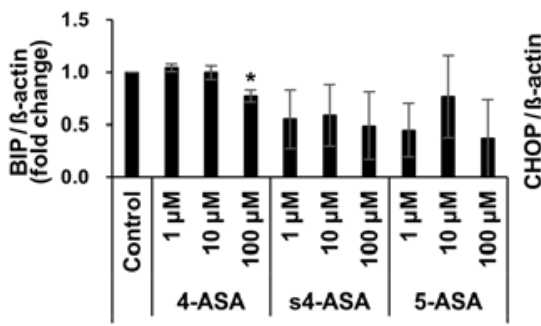

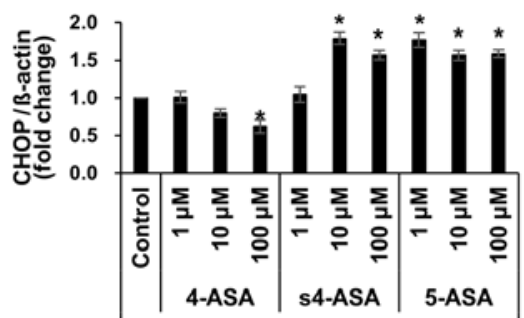

NS
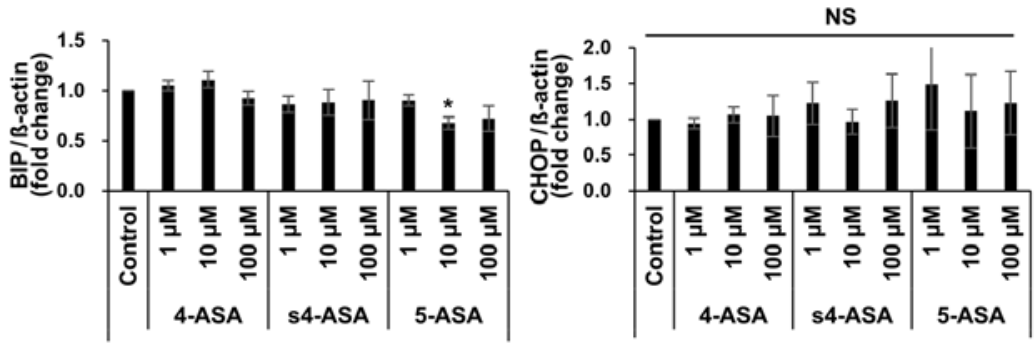

D

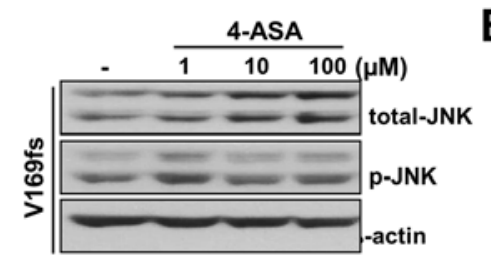

E
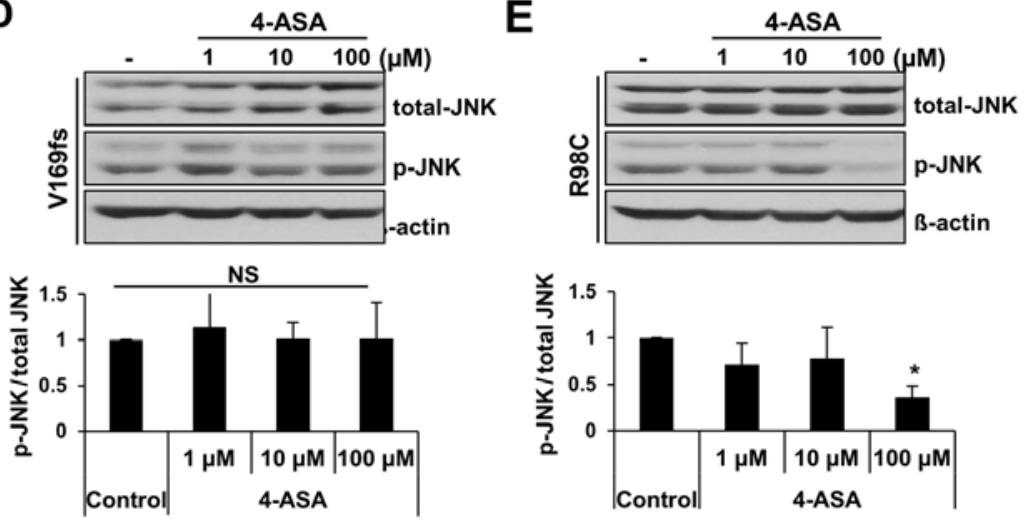

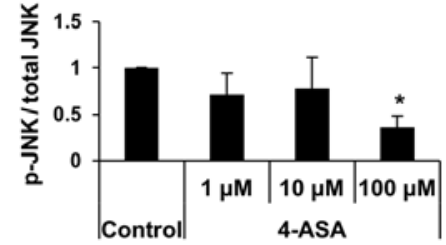

Figure 4. Effects of ASAs on the reduction of ER stress induced by MPZ mutant expression. (A and B) Western blot analysis indicating changes in ER stress markers (BiP and CHOP) following treatment with 3 different concentrations of each ASA in RT4 cells expressing MPZ (A) V169fs and (B) MPZ R98C. Quantitative analyses of western blot analysis data were performed for $\mathrm{BiP}$ and $\mathrm{CHOP}\left(\mathrm{n}=3 ;{ }^{*} \mathrm{P}<0.05\right.$; NS, not significant vs. control). (C) Immunocytochemistry to detect an ER marker (PDI) in cells overexpressing GFP-tagged wild-type MPZ and the V169fs mutant, which indicates that ASA treatment relieves the retention of MPZ V169fs in the ER compartment in RT4 cells ( $\mathrm{n}=3$; scale bar, $20 \mu \mathrm{m}$ ). (D and E) Western blot analysis showing changes in phosphorylated JNK and total JNK levels following treatment with 3 different concentrations of 4-ASA in RT4 cells expressing either (D) V169fs or (E) R98C mutant MPZ. Quantitative analyses of western blot analysis data were performed for the ratio of phosphorylated JNK to total JNK in MPZ V169fs or R98C (n=3; $\mathrm{P}<0.05$; NS, not significant vs. control). MPZ, myelin protein zero; ASA, aminosalicylic acid.

V169fs-expressing RT4 cells, but not in MPZ R98C-expressing cells; iii) 4-ASA released V169fs protein retained in the ER; and iv) 4-ASA decreased the level of p-JNK, which is involved in apoptosis, in R98C protein-expressing RT4 cells, but not in MPZ V169fs-expressing cells.

The structure of MPZ proteins is favorable to be retained in the ER, thus triggering the unfolded protein response (UPR), which can be assessed by UPR markers (BiP and CHOP) (7). Our data support previous evidence that both the V169fs and R98C mutants similarly induced the UPR and ER stress in vitro and in vivo, respectively $(10,21)$. Furthermore, the expression of the V169fs mutant significantly elevated the levels of both ER stress markers compared to expression of wild-type protein, whereas the expression of the R98C mutant caused a significant increase only in the level of CHOP. We hypothesized that the pathological mechanism of V169fs mutant protein involves the retention of mutant proteins in the ER causing an elevation of BiP, which consequently activates downstream effectors (CHOP) and eventually leads to Schwann cell apoptosis. On the other hand, the R98C mutant did not result in this particular cellular phenotype. Based on the evidence, the 2 mutants led to protein structural changes during post-translational modification and caused ER stress in a different manner.

Both MPZ mutants do not seem to share the apoptotic mechanism. According to previous studies, the expression of R98C and S63C mutant proteins did not cause the recovery of Schwann cell apoptosis in the absence of CHOP expression in vivo, although CHOP is important for the demyelination of MPZ mutant nerve cells $(21,23)$. It was also previously demonstrated that R98C mutant protein induced Schwann cell death and UPR activation in the ER via activation of the IRE1 $\alpha / \mathrm{JNK}$ pathway instead of the PERK/CHOP pathway (21). On the other hand, there is no evidence regarding the pathological mechanisms of action of MPZ V169fs protein in Schwann cells, except that patients carrying this frameshift mutation develop more severe neuropathy phenotypes than CMT $(10,33)$. Based on the data of this study, the two different mutations cause different patterns of ER stress and different mechanisms for apoptosis. Although 4-ASA treatment $(100 \mu \mathrm{M})$ resulted in 
A
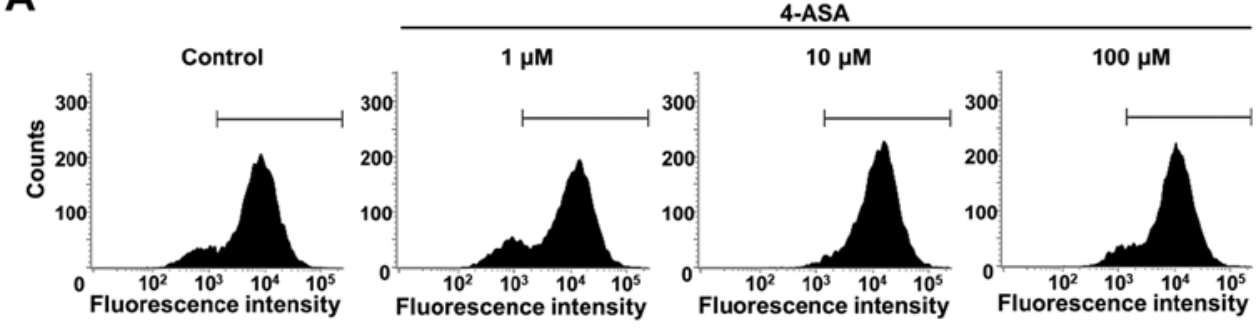

B
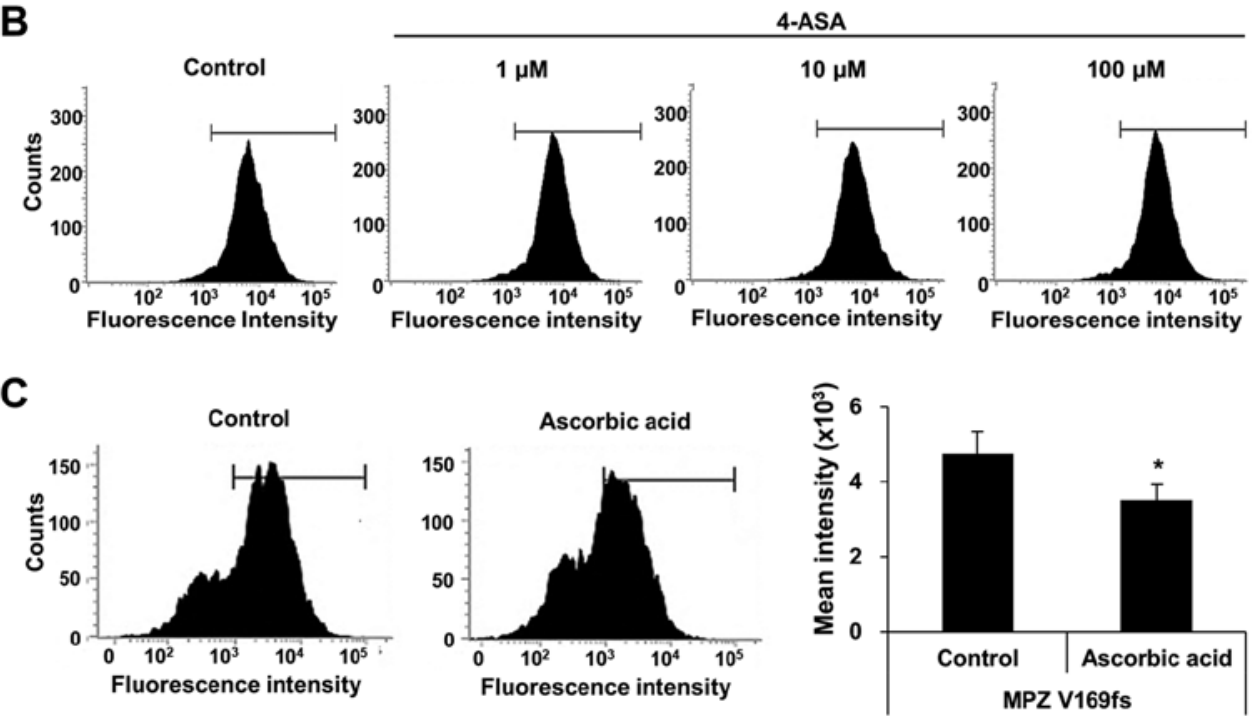

C

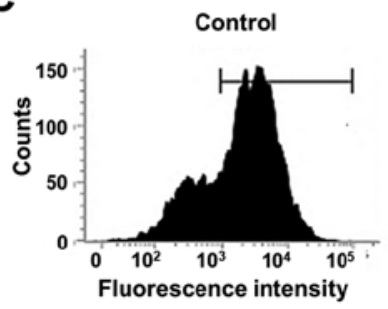

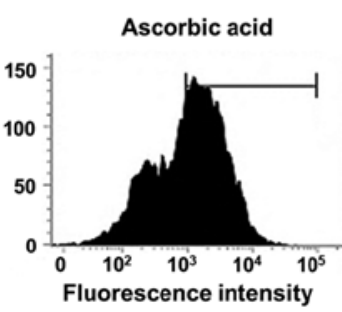

MPZ V169fs
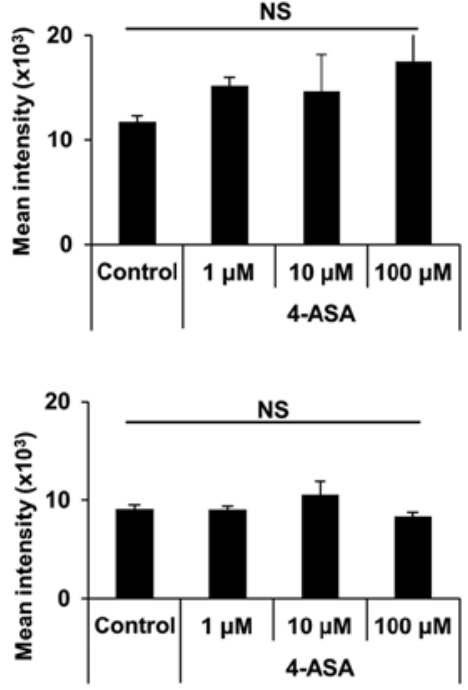

Figure 5. Effects of ASAs and ascorbic acid on the increased ROS levels induced by expression of mutant MPZ proteins. (A) Treatment with 3 different concentrations of 4-ASA in MPZ V169fs-overexpressing RT4 cells did not alter the intracellular ROS levels ( $\mathrm{n}=3$; NS, not significant). (B) DCFDA assay indicating that treatment with 3 different concentrations of 4-ASA in RT4 cells overexpressing MPZ R98C did not alter intracellular ROS levels (n=3; NS, not significant). (C) Treatment of MPZ V169fs-overexpressing RT4 cells with ascorbic acid resulted in a significant reduction of intracellular ROS levels (n=3; $\left.{ }^{*} \mathrm{P}<0.05\right)$. MPZ, myelin protein zero; ASA, aminosalicylic acid; ROS, reactive oxygen species.

significant reduction of apoptosis determined by using cleaved caspase-3 staining, both the BiP and CHOP levels were only significantly reduced in V169fs mutant-expressing cells and p-JNK levels were only reduced in the R98C mutant-expressing cells. Owing to the diverse function of each MPZ mutant protein in Schwann cells, it is necessary to elucidate the pathological mechanisms of individual mutations in order to devise treatment strategies for patients with each pathology as unique gain-of-function mechanisms are involved in CMT and DSN.

Recently, curcumin has been suggested as a promising therapeutic candidate for the treatment of CMT (34), as it allows misfolded proteins to traverse within the ER to the plasma membrane, thereby reducing cytotoxicity (35-37). However, it must be modified for clinical use due to its instability, low efficacy and insolubility (33). From the view of the drug development process, the repositioning of an established drug is a strong alternative option. ASAs are used clinically as anti-inflammatories and antibiotics. In this study, we evaluated the efficacy of ASAs and found that only 4-ASA treatment was effective in alleviating ER stress and reducing apoptosis. Although treatment with the other ASAs (s4-ASA and 5-ASA) resulted in similar outcomes in terms of increasing Schwann cell viability, they did not bring about a reduction in the CHOP level, which was elevated by expression of mutant MPZ proteins. As MPZ mutants elevated ER stress by increasing ROS levels, we measured tje intracellular ROS levels following treatment with 4-ASA; 4-ASA is known to reduce the concentration of free radicals in a manner mediated by nuclear factor- $\kappa \mathrm{B}$ inhibition $(18,19)$. Although 4-ASA reduced the intracellular ROS levels induced by manganese neurotoxicity (20), it seems that 4-ASA could not suppress the MPZ mutant-driven ROS level. These data suggest that 4-ASA may be involved in other pathways of the apoptotic cell death that was caused by the UPR-mediated ER stress in Schwann cells.

In conclusion, in this study, we demonstrated that treatment with 4-ASA reduced the ER stress and SC death caused by 2 different MPZ mutants. However, the treatment efficacy was limited and the mode of action was not clearly revealed. Thus, ASAs need to be further developed to enhance the therapeutic efficacy for the treatment of CMT or DSN. In addition, enhancing the in vitro and in vivo model by the generation of point mutations at the endogenous MPZ using CRISPR/Cas9 technique may be helpful for the better understanding of the MPZ mutations-mediated pathogenesis and the mode-of-action of ASAs. Thus far, there is no treatment available that affects the course of progression of CMT. With an eye towards identifying small molecules or drugs for CMT treatment, 4-ASA warrants further investigationr as a treatment option for CMT or DSN.

\section{Acknowledgements}

Not applicable. 


\section{Funding}

The present study was supported by the Korean Health Technology R\&D Project, Ministry of Health and Welfare (HI14C3484 and HI16C0426) and by the National Research Foundation of Korea (NRF) grants funded by the Korean government, MSIP (NRF-2016R1A5A2007009, NRF-2017R1A2B2004699 and NRF-2018R1A4A1024506) and MOE (2016R1D1A1B03932630).

\section{Availability of data and materials}

The datasets used and/or analyzed during the current study are available from the corresponding author on reasonable request.

\section{Authors' contributions}

EHC and WMM designed and performed the experiments, interpreted the data, and wrote the manuscript. HMD and JSL collected and analyzed data. HTP analyzed and interpreted data. BOC and YBH generated the study concept and design, drafted the manuscript, and provided study supervision. All authors read and approved the final manuscript.

\section{Ethics approval and consent to participate}

Not applicable.

\section{Patient consent for publication}

Not applicable.

\section{Competing interests}

The authors declare that they have no competing interests.

\section{References}

1. Lemke G: Molecular biology of the major myelin genes. Trends Neurosciences 9: 266-270, 1986.

2. Sutcliffe JG: The genes for myelin revisited. Trends Genet 4: 211-213, 1988.

3. Patzig J, Jahn O, Tenzer S, Wichert SP, de Monasterio-Schrader P, Rosfa S, Kuharev J, Yan K, Bormuth I, Bremer J, et al: Quantitative and integrative proteome analysis of peripheral nerve myelin identifies novel myelin proteins and candidate neuropathy loci. J Neurosci 31: 16369-16386, 2011.

4. Warner LE, Hilz MJ, Appel SH, Killian JM, Kolodry EH, Karpati G, Carpenter S, Watters GV, Wheeler C, Witt D, et al: Clinical phenotypes of different MPZ (P0) mutations may include charcot-marie-tooth type $1 \mathrm{~B}$, dejerine-sottas, and congenital hypomyelination. Neuron 17: 451-460, 1996.

5. Mandich P, Mancardi GL, Varese A, Soriani S, Di Maria E, Bellone E, Bado M, Gross L, Windebank AJ, Ajmar F and Schenone A: Congenital hypomyelination due to myelin protein zero Q215X mutation. Ann Neurol 45: 676-678, 1999

6. Murakami T, Garcia CA, Reiter LT and Lupski JR: Charcot-marie-tooth disease and related inherited neuropathies. Medicine (Baltimore) 75: 233-250, 1996.

7. Wrabetz L, D'Antonio M, Pennuto M, Dati G, Tinelli E, Fratta P, Previtali S, Imperiale D, Zielasek J, Toyka K, et al: Different intracellular pathomechanisms produce diverse myelin protein zero neuropathies in transgenic mice. J Neurosci 26: 2358-2368, 2006.

8. Tobler AR, Notterpek L, Naef R, Taylor V, Suter U and Shooter EM: Transport of Trembler-J mutant peripheral myelin protein 22 is blocked in the intermediate compartment and affects the transport of the wild-type protein by direct interaction. J Neurosci 19: 2027-2036, 1999.
9. Sancho S, Young P and Suter U: Regulation of Schwann cell proliferation and apoptosis in PMP22-deficient mice and mouse models of charcot-marie-tooth disease type 1A. Brain 124: 2177-2187, 2001.

10. Khajavi M, Inoue K, Wiszniewski W, Ohyama T, Snipes GJ and Lupski JR: Curcumin treatment abrogates endoplasmic reticulum retention and aggregation-induced apoptosis associated with neuropathy-causing myelin protein zero-truncating mutants. Am J Hum Genet 77: 841-850, 2005.

11. Myers JK, Mobley CK and Sanders CR: The peripheral neuropathy-linked trembler and trembler-J mutant forms of peripheral myelin protein 22 are folding-destabilized. Biochemistry 47: 10620-10629, 2008.

12. Sakakura M, Hadziselimovic A, Wang Z, Schey KL and Sanders CR: Structural basis for the trembler-J phenotype of charcot-marie-tooth disease. Structure 19: 1160-1169, 2011.

13. Rutkowski DT and Kaufman RJ: A trip to the ER: Coping with stress. Trends Cell Biol 14: 20-28, 2004.

14. Welihinda AA and Kaufman RJ: The unfolded protein response pathway in Saccharomyces cerevisiae. Oligomerization and trans-phosphorylation of Ire1p (Ern1p) are required for kinase activation. J Biol Chem 271: 18181-18187, 1996.

15. Hetz C and Mollereau B: Disturbance of endoplasmic reticulum proteostasis in neurodegenerative diseases. Nat Rev Neurosci 15: 233-249, 2014.

16. Mitchison DA: Role of individual drugs in the chemotherapy of tuberculosis. Int J Tuberc Lung Dis 4: 796-806, 2000.

17. Daniel F, Seksik P, Cacheux W, Jian R and Marteau P: Tolerance of 4-aminosalicylic acid enemas in patients with inflammatory bowel disease and 5-aminosalicylic-induced acute pancreatitis. Inflamm Bowel Dis 10: 258-260, 2004.

18. Joshi R, Kumar S, Unnikrishnan M and Mukherjee T: Free radical scavenging reactions of sulfasalazine, 5-aminosalicylic acid and sulfapyridine: Mechanistic aspects and antioxidant activity. Free Radic Res 39: 1163-1172, 2005.

19. Patole J, Shingnapurkar D, Padhye S and Ratledge C: Schiff base conjugates of $\mathrm{p}$-aminosalicylic acid as antimycobacterial agents. Bioorg Med Chem Lett 16: 1514-1517, 2006.

20. Yoon H, Lee GH, Kim DS, Kim KW, Kim HR and Chae HJ: The effects of 3, 4 or 5 amino salicylic acids on manganese-induced neuronal death: ER stress and mitochondrial complexes. Toxicol In Vitro 25: 1259-1268, 2011

21. Saporta MA, Shy BR, Patzko A, Bai Y, Pennuto M, Ferri C, Tinelli E, Saveri P, Kirschner D, Crowther M, et al: MpzR98C arrests Schwann cell development in a mouse model of early-onset charcot-marie-tooth disease type 1B. Brain 135: 2032-2047, 2012.

22. Grandis M, Vigo T, Passalacqua M, Jain M, Scazzola S, La Padula V, Brucal M, Benvenuto F, Nobbio L, Cadoni A, et al: Different cellular and molecular mechanisms for early and late-onset myelin protein zero mutations. Hum Mol Genet 17: 1877-1889, 2008.

23. Pennuto M, Tinelli E, Malaguti M, Del Carro U, D'Antonio M, Ron D, Quattrini A, Feltri ML and Wrabetz L: Ablation of the UPR-mediator CHOP restores motor function and reduces demyelination in charcot-marie-tooth 1B mice. Neuron 57: 393-405, 2008.

24. Haynes CM, Titus EA and Cooper AA: Degradation of misfolded proteins prevents ER-derived oxidative stress and cell death. Mol Cell 15: 767-776, 2004.

25. Xue X, Piao JH, Nakajima A, Sakon-Komazawa S, Kojima Y, Mori K, Yagita H, Okumura K, Harding $\mathrm{H}$ and Nakano H: Tumor necrosis factor alpha (TNFalpha) induces the unfolded protein response (UPR) in a reactive oxygen species (ROS)-dependent fashion, and the UPR counteracts ROS accumulation by TNFalpha. J Biol Chem 280: 33917-33925, 2005.

26. Williams C, Panaccione R, Ghosh S and Rioux K: Optimizing clinical use of mesalazine (5-aminosalicylic acid) in inflammatory bowel disease. Therap Adv Gastroenterol 4: 237-248, 2011.

27. Fox W, Ellard GA and Mitchison DA: Studies on the treatment of tuberculosis undertaken by the british medical research council tuberculosis units, 1946-1986, with relevant subsequent publications. Int J Tuberc Lung Dis 3 (Suppl 2): S231-S279, 1999.

28. Urano F, Wang X, Bertolotti A, Zhang Y, Chung P, Harding HP and Ron D: Coupling of stress in the ER to activation of JNK protein kinases by transmembrane protein kinase IRE1. Science 287: 664-666, 2000.

29. Cao SS and Kaufman RJ: Endoplasmic reticulum stress and oxidative stress in cell fate decision and human disease. Antioxid Redox Signal 21: 396-413, 2014. 
30. Fukumura H, Sato M, Kezuka K, Sato I, Feng X, Okumura S, Fujita T, Yokoyama U, Eguchi H, Ishikawa Y and Saito T: Effect of ascorbic acid on reactive oxygen species production in chemotherapy and hyperthermia in prostate cancer cells. J Physiol Sci 62: 251-257, 2012

31. Peng Y, Kwok KH, Yang PH, Ng SS, Liu J, Wong OG, He ML, Kung HF and Lin MC: Ascorbic acid inhibits ROS production, NF-kappa B activation and prevents ethanol-induced growth retardation and microencephaly. Neuropharmacology 48: 426-434, 2005.

32. Shy ME, Jáni A, Krajewski K, Grandis M,Lewis RA, Li J, Shy RR, Balsamo J, Lilien J, Garbern JY and Kamholz J: Phenotypic clustering in MPZ mutations. Brain 127: 371-384, 2004.

33. Patzko A, Bai Y, Saporta MA, Katona I, Wu X, Vizzuso D, Feltri ML, Wang S, Dillon LM, Kamholz J, et al: Curcumin derivatives promote schwann cell differentiation and improve neuropathy in R98C CMT1B mice. Brain 135: 3551-3566, 2012.

34. Okamoto Y, Pehlivan D, Wiszniewski W, Beck CR, Snipes GJ, Lupski JR and Khajavi M: Curcumin facilitates a transitory cellular stress response in trembler-J mice. Hum Mol Genet 22: 4698-4705, 2013.
35. Egan ME, Pearson M, Weiner SA, Rajendran V, Rubin D, Glockner-Pagel J, Canny S, Du K, Lukacs GL and Caplan MJ: Curcumin, a major constituent of turmeric, corrects cystic fibrosis defects. Science 304: 600-602, 2004.

36. Vasireddy V, Chavali VR, Joseph VT, Kadam R, Lin JH, Jamison JA, Kompella UB, Reddy GB and Ayyagari R: Rescue of photoreceptor degeneration by curcumin in transgenic rats with P23H rhodopsin mutation. PLoS One 6: e21193, 2011.

37. Wang Y, Xiao J, Zhou H, Yang S, Wu X, Jiang C, Zhao Y, Liang D, Li X and Liang G: A novel monocarbonyl analogue of curcumin, (1E,4E)-1,5-bis(2,3-dimethoxyphenyl) penta-1,4-dien-3-one, induced cancer cell H460 apoptosis via activation of endoplasmic reticulum stress signaling pathway. J Med Chem 54: 3768-3778, 2011.

(i) (5) This work is licensed under a Creative Commons Attribution-NonCommercial-NoDerivatives 4.0 International (CC BY-NC-ND 4.0) License. 